\title{
Correction: MiR-451 as a new tumor marker for gastric cancer
}

\section{Yong Shen ${ }^{1,2}$, Jiao-Mei Gong ${ }^{3}$, Li-Li Zhou ${ }^{4}$ and Jia-He Sheng ${ }^{2}$}

${ }^{1}$ Department of Pathology and Pathophysiology, Medical School of Southeast University, Nanjing, Jiangsu, China

${ }^{2}$ Department of Clinical Laboratory, Affiliated Cancer Hospital of Zhengzhou University, Zhengzhou, Henan, China

${ }^{3}$ Department of Clinical Laboratory, The Second Affiliated Hospital of Zhengzhou University, Zhengzhou, Henan, China

${ }^{4}$ Department of Hepatobiliary Surgery, Affiliated Cancer Hospital of Zhengzhou University, Zhengzhou, Henan, China

\section{Published: October 29, 2019}

Copyright: Shen et al. This is an open-access article distributed under the terms of the Creative Commons Attribution License 3.0 (CC BY 3.0), which permits unrestricted use, distribution, and reproduction in any medium, provided the original author and source are credited.

This article has been corrected: The authors' affiliation information was listed incorrectly. The proper affiliations are as follows:

${ }^{1}$ Department of Pathology and Pathophysiology, Medical School of Southeast University, Nanjing, Jiangsu, China

${ }^{2}$ Department of Clinical Laboratory, Affiliated Cancer Hospital of Zhengzhou University, Zhengzhou, Henan, China

${ }^{3}$ Department of Clinical Laboratory, The Second Affiliated Hospital of Zhengzhou University, Zhengzhou, Henan, China

${ }^{4}$ Department of Hepatobiliary Surgery, Affiliated Cancer Hospital of Zhengzhou University, Zhengzhou, Henan, China

Original article: Oncotarget. 2017; 8:56542-56545. https://doi.org/10.18632/oncotarget.17239 